\title{
Far-IR to Submm SEDs for local galaxies: Herschel, Planck and the HRS
}

\author{
David L Clements ${ }^{1}$ and \\ The Herschel Reference Sample Team \\ ${ }^{1}$ Astrophysics Group, Imperial College, Blackett Laboratory, \\ Prince Consort Road, London, SW7 2AZ,UK. \\ email: d.clements@imperial.ac.uk
}

\begin{abstract}
The Herschel Space Observatory and the Planck satellite are providing radical improvements to our knowledge of the spectral energy distributions of galaxies in the far-IR and submm. We here present the results of the first combination of Herschel and Planck fluxes of local galaxies from the Herschel Reference Sample (HRS) survey, covering galaxies at distances between 15 and $25 \mathrm{Mpc}$. This combination provides information on SEDs in eight bands from $60 \mu \mathrm{m}$, using IRAS, to $1.4 \mathrm{~mm}$ using Planck. We apply a similar fitting procedure to this data as applied to the Planck ERCSC-detected nearby galaxies and confirm the result that dust significantly colder than $20 \mathrm{~K}$ is common in local galaxies. It is early days for this kind of study, but it is clear that the new generation of satellites are already adding considerably to our knowledge of the far-IR/submm properties of galaxies.
\end{abstract}

Keywords. galaxies:dust, Herschel Space Observatory, Planck, galaxies:infrared, galaxies:submm

\section{Introduction}

The last 15 years have seen a huge increase in our capability for studying the long wavelength far-IR $(\lambda>100 \mu \mathrm{m})$ and submm spectral energy distributions (SEDs) of galaxies. Where once we would have to rely on IRAS data that cuts off at $100 \mu \mathrm{m}$ and perhaps one or two points at longer wavelengths with large error bars from single element bolometer detectors, such as UKT-14 on JCMT (eg. Clements et al. 1993), we can now extend our coverage to longer wavelength far-IR points by using ISO and Spitzer, while our submm capabilities have been immensely improved by bolometer arrays such as SCUBA and MAMBO. Our capabilities are now once more being boosted, and this time the important wavelength region between 200 and $160 \mu \mathrm{m}$, respectively the longest wavelength ISO and Spitzer bands, and $450 \mu \mathrm{m}$, the longest wavelength band for which significant ground based data is available, is being filled. The Herschel Space Observatory (Pilbratt et al. 2010) and the Planck satellite (Planck Collaboration, 2011a) are each playing their role. Herschel provides detailed sensitive pointed observations from 70 to $500 \mu \mathrm{m}$, with the SPIRE instrument (Griffin et al. 2010) covering 3 bands from 250 to $500 \mu \mathrm{m}$. Planck, meanwhile, is providing all sky coverage in nine bands from $350 \mu \mathrm{m}$ ( $857 \mathrm{GHz}$ in Planck parlance) to $30 \mathrm{GHz}$. This is the first all sky survey for many of these bands, providing especially important constraints on both the cool dust that can be seen in the submm (eg. Planck Collaboration, 2011b) and on free-free and anomalous microwave emission at wavelengths longer than about 1mm (Planck Collaboration, 2011c; Peel et al. 2011).

The full impact of the new capabilities that Herschel and Planck provide has still to arrive, but first results can already be seen in this volume and elsewhere. In this 
contribution we will be looking at the results of combining Herschel and Planck data for nearby galaxies that have been observed as part of the Herschel Reference Survey (HRS; Boselli et al. 2010).

\section{The HRS and the Planck ERCSC}

The HRS (Boselli et al. 2010) is a survey of a statistically complete sample of local galaxies with the Herschel SPIRE instrument. Images of 323 galaxies at 250, 350 and $500 \mu \mathrm{m}$ have been obtained and additional observations to 100 and $160 \mu \mathrm{m}$ have been proposed. The sample is volume limited with distances between 15 and $25 \mathrm{Mpc}$ and covers the whole range of morphological types, from ellipticals to spirals, and environments, from the field to the centre of the Virgo cluster.

The Planck Early Release Compact Source Catalog (ERCSC; Planck Collaboration, 2011d) is one of the first public data releases from the Planck mission. It includes fluxes for all compact sources detected in Planck bands with high reliability (90\% or greater) during the first Planck all sky survey. Of specific interest to the present work is the $857 \mathrm{GHz}$ catalog in the ERCSC since this includes additional band merged fluxes at 545, 353 and $217 \mathrm{GHz}$. It is thus a $350 \mu \mathrm{m}(857 \mathrm{GHz})$ selected sample with additional fluxes at 550,850 and $1400 \mu \mathrm{m}$. This makes it an ideal resource for studying the far-IR to submm SEDs of galaxies (see e.g. Planck Collaboration, 2011b).

We combine the ERCSC and HRS catalogs by searching for all sources in the HRS that appear in the ERCSC $857 \mathrm{GHz}$ catalog. We find 152 matches. Of these, there is a $>1 \sigma$ difference between the Planck $350 \mu \mathrm{m}$ fluxes and the currently available HRS fluxes. This is because the current HRS flux extraction is not determining the fluxes within the large $\left(\sim 5^{\prime}\right)$ Planck beams. Instead it is generated using a much smaller beam, so that extended emission and companion sources are not properly accounted for. The production of HRS fluxes optimised for matching to Planck data is currently underway, but is not yet complete.

\section{HRS \& Planck SEDs}

We combine our HRS and Planck fluxes with the IRAS fluxes for these sources, and use the same Bayesian SED fitting approach that was used in Planck Collaboration (2011b) to fit the SEDs of Planck sources. The model SEDs that we fit are based on the usual empirical $F_{\nu} \propto \nu^{\beta} B_{\nu}(T)$ SED, where $F_{\nu}$ is the flux of the source at frequency $\nu, \beta$ is the emissivity, usually between 1 and $2, \mathrm{~B}_{\nu}$ is a Planckian function and $\mathrm{T}$ is the temperature of the dust. We try fits using a single dust component with $\mathrm{T}$ and $\beta$ as free parameters, two dust components at different temperatures, $\mathrm{T}_{1}$ and $\mathrm{T}_{2}$, and $\beta$ fixed to 2 (eg. Dunne $\&$ Eales 2001), and two components with different temperatures and $\beta$ s. Our Bayesian fitting approach not only allows the best fit and accurate errors on fitted parameters to be calculated, even in the presence of correlated errors in the parameters, but it also allows the Bayesian Evidence (Jaffe 1996; Jaynes 2003) for specific fits and models to be calculated. In this way we can use the Bayes factor to determine which of the models is the better fit for a given object, taking into account the increasing numbers of free parameters where there are more components. There is also the prospect of combining the Bayesian Evidence for all objects for a given fit to determine which model is best at describing the whole sample.

In Fig. 1 we show the results of the single component and two component $\beta=2$ fits for the combined HRS and Planck fluxes. We also show the results of similar fits using IRAS and Planck data alone, and the results of SED fits to IRAS and SCUBA 

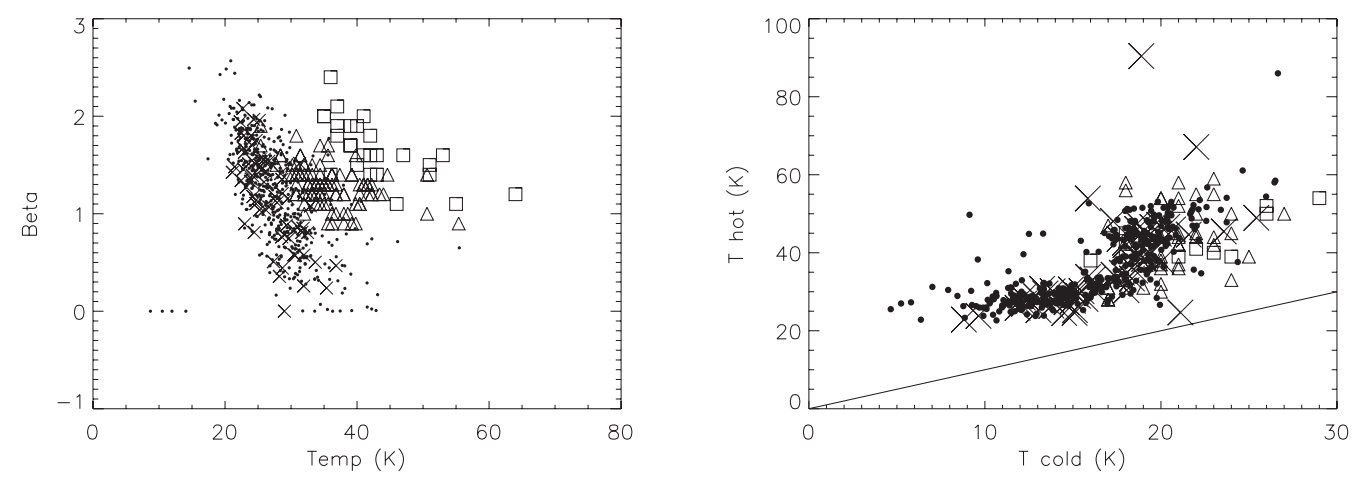

Figure 1. Comparison of the results of parametric fits to the IRAS, HRS and Planck fluxes of the HRS sources (crosses) to similar fits to Planck ERCSC sources (black dots) and to sources observed as part of the SLUGS survey (triangles; Dunne et al. 2000; Dunne \& Eales 2001) and local ULIRGs (Clements et al. 2010).

submm data. As can be seen, the results of our parametric fits to those galaxies where Herschel data is available are broadly similar to the fits obtained using just Planck and IRAS data. We thus confirm the key result on nearby galaxies from the Planck ERCSC (Planck Collaboration, 2011b) that cold dust, with temperatures $<<20 \mathrm{~K}$, is a significant constituent of the interstellar medium of galaxies.

\section{Discussion and Conclusions}

We have confirmed the earlier results on these galaxies obtained from just Planck and IRAS data that cold dust with $\mathrm{T}<<20 \mathrm{~K}$ is an important constituent of the interstellar medium of nearby galaxies, but there is still much to be done. With the resolved Herschel maps of these sources it will be possible to determine the spatial distribution of different dust components, warm and cold. Meanwhile, once the beam-matched photometry for HRS galaxies is complete, the larger sample size will allow us to cross correlate dust properties to other galaxy properties such as environment, morphology and star formation history. More broadly, the overall methodology used here establishes a framework in which a wide range of SED models, physical as well as parametric, may be tested against the data. Given a large enough sample of galaxies we will be able to draw conclusions about the applicability of these different models using Bayesian Evidence and Bayesian model selection. In this way we hope that the current large range of different models for dust SEDs can be quantitatively compared, and an optimum set of models will emerge.

\section{Acknowledgements}

We would like to thank Laure Cisela for her work on the HRS photometry used in this paper and Andrew Jaffe for the provision of the Bayesian SED fitting code. This work is funded in part by STFC. This work is based on observations obtained with Planck, an ESA science mission with instruments and contributions directly funded by ESA Member States, NASA, and Canada. The development of Planck has been supported by: ESA; CNES and CNRS/INSU-IN2P3-INP (France); ASI, CNR, and INAF (Italy); NASA and DoE (USA); STFC and UKSA (UK); CSIC, MICINN and JA (Spain); Tekes, AoF and CSC (Finland); DLR and MPG (Germany); CSA (Canada); DTU Space (Denmark); SER/SSO (Switzerland); RCN (Norway); SFI (Ireland); FCT/MCTES (Portugal); and DEISA (EU). Herschel is an ESA space observatory with science instruments provided by European-led Principal Investigator consortia and with important participation from 
NASA. SPIRE has been developed by a consortium of institutes led by Cardiff University (UK) and including Univ. Lethbridge (Canada); NAOC (China); CEA, LAM (France); IFSI, Univ. Padua (Italy); IAC (Spain); Stockholm Observatory (Sweden); Imperial College London, RAL, UCL-MSSL, UKATC, Univ. Sussex (UK); and Caltech, JPL, NHSC, Univ. Colorado (USA). This development has been supported by national funding agencies: CSA (Canada); NAOC (China); CEA, CNES, CNRS (France); ASI (Italy); MCINN (Spain); SNSB (Sweden); STFC (UK); and NASA (USA).

\section{References}

Boselli, A. et al. 2010, PASP, 122, 261

Clements, D. L., Andreani, P., \& Chase, S. T. 1993, MNRAS, 261, 299

Clements, D. L., Dunne, L., \& Eales, S. 2010, MNRAS, 403, 274

Dunne, L. \& Eales, S. A. 2001, MNRAS, 327, 697

Dunne, L. et al. 2000, MNRAS, 315, 115

Griffin, M. J. et al. 2010, A\& A, 518, L3

Jaffe, A. 1996, ApJ, 471, 24

Jaynes, E. 2003, Probability Theory: the Logic of Science ed. L. Bretthorst (Cambridge University Press)

Peel, M. W. et al. 2011, MNRAS, 416, L99

Pilbratt, G. L. et al. 2010, A\&A, 518, L1

Planck Collaboration 2011a, A\&SA 536, A1

Planck Collaboration 2011b, $A \mathscr{E} A$, 536, A16

Planck Collaboration 2011c, $A \mathscr{G} A$, 536, A17

Planck Collaboration 2011d, AESA, 536, A7

\section{Discussion}

MAdDen: The data you are using, especially the Planck data, contains dust + CO + free- free/synchrotron. When you perform your Bayesian fits, can you add a radio continuum (free-free, synchrotron) component, so that your resultant B-T solutions are really applicable to the dust? Otherwise your results for B-T cannot be related to dust temperature or dust emissivity.

Clements: We don't add extra components like CO, free- free, synchrotron at this stage but it would certainly be possible. At the moment we do not push to the long wavelengths where these terms are large. But for some bright sources this will be possible and necessary in the end. 